Nervenarzt 2020 $91: 1017-1024$ https://doi.org/10.1007/s00115-020-00990-1 Online publiziert: 15 . September 2020 (c) Springer Medizin Verlag $\mathrm{GmbH}$, ein Teil von Springer Nature 2020

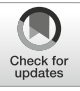

\author{
U. Kluge ${ }^{1,2} \cdot$ M. C. Aichberger ${ }^{1,2} \cdot$ E. Heinz' $\cdot$ C. Udeogu-Gözalan ${ }^{3} \cdot$ D. Abdel-Fatah ${ }^{1,2}$ \\ ${ }^{1}$ Klinik für Psychiatrie und Psychotherapie, Charité Campus Mitte, Charité Universitätsmedizin Berlin, \\ Berlin, Deutschland \\ ${ }^{2}$ Berliner Institut für Empirische Integrations- und Migrationsforschung (BIM), Humboldt-Universität zu \\ Berlin, Berlin, Deutschland \\ ${ }^{3}$ Psychotherapeutische Hochschulambulanz für Säuglinge, Kinder, Jugendliche und Erwachsene, Ludwig- \\ Maximilians-Universität, München, Deutschland
}

\title{
Rassismus und psychische Gesundheit
}

Anwendung zu Beginn 2020 ein Schwerpunktheft [47]. Die Autor*innen diskutieren darin die gesellschaftlichen, biographischen und psychischen Langzeitfolgen rechter und rassistisch motivierter Gewalt und formulieren als zentrales Anliegen die Notwendigkeit, die Stimmen der von rechter Gewalt Betroffenen in der Öffentlichkeit hörbar zu machen. Diese Erfahrungsberichte, wie sie beispielsweise in der Podcast-Serie von NSU Watch und VBRG e.V. „Vor Ort - gegen Rassismus, Antisemitismus und rechte Gewalt" (siehe: https://www. verband-brg.de/podcast/) zusammengetragen wurden, verweisen nicht nur auf die Notwendigkeit der gesamtgesellschaftlichen Solidarität mit den Opfern, sondern sind zugleich Zeugnisse der Auswirkungen rassistischer Gewalt auf die Erfahrungswelten und damit die psychische Gesundheit der Opfer.

Für eine differenzierte Debatte in der Psychiatrie und zum Umgang mit von Rassismus Betroffenen werden wir im folgenden Beitrag drei Stränge des aktuellen Diskussionsstandes genauer beleuchten:

I. Rassismusdiskurse und eine kritische Betrachtung der Begriffe „Rasse“ und „race“,

II. die Auswirkungen von Rassismus und Diskriminierung auf Wohlbefinden und psychische Gesundheit und abschließend

III. Umgang mit Rassismus und Diskriminierung in Versorgungsstrukturen.

\section{Rassismusdiskurse und eine kritische Betrachtung der Begriffe „Rasse" und „race“}

Nach dem Ende des Zweiten Weltkrieges etablierte sich in Europa und Deutschland der Eindruck (oder auch die Hoffnung), dass „rassi(sti)sche“ Kategorisierungen und Etikettierungen der Vergangenheit angehören (vgl. [48]). Es mag daher verwundern, dass der Begriff „Rasse" bis heute im deutschen Grundgesetz verankert ist [23]. Neben „Abstammung“ und „Sprache“ wird er erwähnt - in einer Reihe von Kategorien, anhand derer Menschen in Deutschland nicht diskriminiert werden sollen. Es scheint also nötig, noch einmal eine historische und zeitgemäße Verortung des Begriffes vorzunehmen.

In der Anthropologie, die sich als naturwissenschaftlicher Zweig aus der Ethnographie entwickelt hatte, beschäftigte man sich bereits im 17. Jahrhundert mit dem Konzept der „Rasse“ zur Klassifizierung von Menschen (vgl. [31]). Das naturwissenschaftliche Konzept der „Rassen“ diente vor allem im 19. und 20. Jahrhundert dazu, das in Europa vorherrschende Klischee einer vermeintlich überlegenen weißen "Rasse“ wissenschaftlich $\mathrm{zu}$ untermauern. Politisch folgte daraus eine Vermischung von Nationalismus und Rassismus mit den bekannten verhängnisvollen Konsequenzen. Die Hypothese lautete, dass es weltweit zumindest drei „Menschenrassen“ (Europäer, Asiaten und Afrikaner) unterschiedlichen örtlichen und zeit- 
lichen Ursprungs gäbe, die eindeutig voneinander abgrenzbar seien. Diese Wahrnehmung fungierte auch als ideologische Säule für den Kolonialismus (vgl. [49]).

Heutige Theorien zu Wanderungsbewegungen der Menschen gehen demgegenüber aber davon aus, dass der Homo sapiens vor etwa 200.000 Jahren in Afrika entstand und sich von dort über Wanderungsbewegungen nach Europa, Asien und schließlich Amerika ausgebreitet hat. Dementsprechend findet sich die höchste genetische Varianz bei Menschen südlich der Sahara in Afrika, wo die ursprüngliche Population vermutet wird [20, 51]. Im Zuge der Wanderungsbewegungen nimmt die genetische Varianz ab. Das ist offenbar darauf zurückzuführen, dass die einzelnen Individuen in eher kleinen Menschengruppen, die die Wanderung antraten, nur einen Teil der Varianz des ursprünglichen Genpools „mitbrachten“ (vgl. [7, 41]). Das heißt, dass die Varianz zwischen zwei Personen südlich der Sahara größer sein kann als zwischen einer Person aus Afrika und einer zweiten aus Asien oder Europa [78].

Es handelt sich dabei allerdings um Unterschiede in der Häufigkeit der Ausprägung von Genvarianten, also von Allelen und ihren Kombinationen und nicht um qualitativ „andersartige“ Gene oder um Allele, die ausschließlich in einer „Rasse“vorkämen [16, 73]. Da der Begriff der „Rasse“ aber auf kategoriale Unterschiede zwischen menschlichen Populationen hinweist, wurde er als biologisch fehlleitend abgelehnt $[3,59]$. Stattdessen wird auf die genetische und phänotypische Vielfalt und die gleitenden Übergänge zwischen verschiedenen Populationen verwiesen ([51]; Livingston 1960).

\section{》) Das Konzept der "Rasse" ist nicht Voraussetzung, sondern Folge von Rassismus}

Dieses Verständnis konstatiert auch die Jenaer Erklärung, die von Forscher*innen aus Zoologie und Anthropologie an der Friedrich-SchillerUniversität Jena im Jahr 2019 zum Anlass des 100. Todestages Ernst Haeckels verabschiedet wurde und die darlegt, dass das Konzept der „Rasse“ nicht die Voraussetzung, sondern die Folge von Rassismus ist [29].

Folgen wir diesem Verständnis, können wir mit Delgado und Stefanic davon ausgehen, dass es sich bei den Begriffen „Rasse“ und „Rassen“ um sozial konstruierte Macht- und Dominanzkategorisierungen handelt [25, S. 7]. So gesehen umfasst Rassismus dann viel mehr als individuelle Vorurteile, Stereotypen und daraus resultierendes Verhalten, sondern ist eingeschrieben in soziale und politische Strukturen und trägt dazu bei, dass rassistisch determinierte Ungleichheit vielfach als natürlich (biologisch) gegebener Prozess konstruiert und wahrgenommen wird und nicht als Ergebnis von Machtund Herrschaftsstrukturen [13].

Sozialkonstruktivistische Konzeptionen der "Rassifizierung“ und des dadurch entstehenden Begriffs der „Rasse ${ }^{\text {“ }}$ unterstreichen hingegen die sozial determinierte hierarchische Einteilung von Menschen in Gruppen basierend auf Phänotypen oder anderen sichtbaren Merkmalen als Teil eines Systems, welches „Rassen“-basierte Ungleichheit und Hierarchie rechtfertigt. So zeigt sich beispielsweise die strukturelle Benachteiligung von Schwarzen in den USA anhand der ausgeprägten Disparitäten im sozioökonomischen Bereich zwischen schwarzen und weißen Amerikanern [28]. Aber auch für Deutschland zeigen Studien für diverse Lebensbereiche Ungleichheiten, die sich entlang der $\mathrm{Zu}$ schreibungen einer anderen ethnischen Herkunft, Hautfarbe oder Religion ergeben. Aktuelle empirische Studien weisen darauf hin, dass entsprechend kategorisierte Gruppen rassistischer Diskriminierung auf dem Wohnungsmarkt [1], im Bildungsbereich $[9,11]$, auf dem Arbeitsmarkt [50] und im Gesundheitssystem [5] ausgesetzt sind.

Im angloamerikanischen Raum hat sich eine kritische Bewegung etabliert, die das soziale Konstrukt der "race“ aktiv nutzt, um die Personen nennbar und sichtbar zu machen, die besonders von derartiger rassistischer Diskriminierung und Ausgrenzung betroffen sind, ohne dabei von biologischen Kategorien auszugehen. Im medizinischen Kontext überwiegt allerdings beim Ge- brauch dieses Begriffs die traditionelle Kategorisierung in biologisch verstandene Gruppen (Pubmed-Abfrage am 21.07.2020: 77.953 Ergebnisse zu „race/ genetic“, $1696 \mathrm{zu}$ „race/social construct“ und $6623 \mathrm{zu}$ "race/discrimination“). Die Übertragung des Begriffs in den deutschen medizinischen Kontext läuft damit Gefahr, biologisch falsche Dichotomisierungen, die traditionell mit dem Begriff der „Rasse“ verbunden waren, zu reproduzieren [42].

\section{》) Rassismus ist ein historisch gewachsenes Phänomen}

Das Dilemma besteht darin, sich einerseits von dem Konstrukt „Rasse“ als biologische Kategorie zu verabschieden, zugleich aber dessen Wirkmacht anzuerkennen. Guillaumin formuliert dazu: "Rassen gibt es nicht und doch töten sie“ [35, S. 7]. Es scheint daher nötig, zu realisieren, dass Rassismus ein historisch gewachsenes Phänomen ist, welches Machtstrukturen ausdrückt, in deren Folge betroffene Gruppen strukturell benachteiligt sind [12, 45, 75].

Eine solche Perspektive ermöglicht es, Rassismus sowohl auf der individuellen als auch strukturellen Ebene als eine zentrale Facette von Gesundheit und Ungleichheit ins Zentrum empirischer Forschung zu rücken [30]. Dies erlaubt es $\mathrm{zu}$ untersuchen, wie über „Rasse“-Zuschreibungen Mitglieder der Gesellschaft aufgrund ihrer individuellen körperlichen Merkmale kategorisiert und etikettiert werden und wie dies wiederum den Zugang zu Gesundheitsstrukturen und -privilegien beeinflusst [33]. Es gibt jedoch bislang keinen Konsens zur Konzeptualisierung und Operationalisierung von Rassismus in epidemiologischer und medizinischer Forschung [55]. Einen solchen zu etablieren, bleibt eine Herausforderung. Allerdings wurden in den letzten Jahrzehnten Modelle zu den verschiedenen Ebenen von Rassismus entwickelt, um Diskurse und Forschung zu „Rassen“bedingten Ungleichheiten besser strukturieren zu können [36, 45]. 
Rassismus und Diskriminierung - Auswirkungen auf Wohlbefinden und psychische Gesundheit

Es gibt eine Vielzahl an Studien, die zeigen, dass sozial marginalisierte Gruppen besonders von psychischen Belastungen und psychischen Erkrankungen betroffen sind. Personen, die einer ethnischen Minderheit angehören, bzw. wie in Deutschland gefasst, einen Migrationshintergrund haben, gehören in besonderem Maße zu dieser Gruppe [18, 40, 61, 72]. Die spezifischen negativen Auswirkungen von Diskriminierung und Ausgrenzung auf psychische Gesundheit wurden in den letzten Jahrzehnten für diverse soziale und ethnische Gruppen unter Berücksichtigung der jeweiligen Kontexte gezeigt $[2,8,10,65,66,87]$. Dabei ist wichtig zu betonen, dass diesbezüglich sowohl für typische stressassoziierte Belastungsreaktionen [66] als auch für schwere psychische Störungen wie Psychosen Evidenz gefunden wurde [46, 80-82]. Eine umfassende metaanalytische Übersichtsarbeit von Cantor-Graae und Selten [18] wies u. a. auf ein erhöhtes Risiko für psychische Störungen wie Schizophrenie bei Personen mit eigener Migrationserfahrung als auch deren Kindern hin.

\section{》) Strukturelle Diskriminierung beeinflusst die frühe psychische Prägung}

Während Diskriminierung auf der individuellen Ebene in Form alltäglicher Mikroaggressionen [64] bis hin zu auBergewöhnlich belastenden Ereignissen mit potenziell traumatisierender Auswirkung erfolgen und sich individuell krankheitsfördernd auswirken kann, muss zugleich die Bedeutung struktureller Diskriminierung betont werden. Strukturelle Diskriminierung beeinflusst über die Lebensspanne sowohl frühe psychische Prägungen, die Verteilung psychischer Ressourcen, Bildungs- und Arbeitsmarktchancen als auch umweltbezogene Risikofaktoren (z.B. Wohnen, Exposition zu Umweltgiften etc.). Die Diskriminierungsforscherin Nancy
Krieger argumentiert deshalb für ein sozialökologisches Modell für die Analyse von Rassismus und Gesundheit, in welchem vor allem ökonomische und soziale Deprivation, Exposition gegenüber Umweltgiften, soziales Trauma, gesundheitsschädigendes Verhalten in Reaktion auf Diskriminierung, genauso wie gruppenspezifisch zielgerichtetes Marketing und unzureichende medizinische Versorgung Berücksichtigung finden sollen $[52,53]$. Sozialökologische Betrachtungsweisen und Analysen weisen auf ein komplexes Zusammenspiel zwischen individuellen Risikofaktoren und sozialen Rahmenbedingungen über die Lebensspanne hin [62], durch welches manche Gruppen einem höheren Risiko unter Risiko ausgesetzt sind, durch solche Risikofaktoren gefährdet zu werden (,increased risk of risks“; [58]).

\section{》) Rassistische Diskriminierung erhöht das Risiko für eine psychotische Episode}

Eine Vielzahl solcher sozialer Ausschlussmechanismen zeigt sich beispielsweise in Studien zu sozialem Kapital in Migrant ${ }^{*}$ innenpopulationen. Deutlich wird dabei, dass Migrant ${ }^{\star}$ innen zwar häufig über engere soziale Netzwerke innerhalb der eigenen migrantischen Gemeinschaft verfügen, aber ihre Netzwerke in die Majoritätsgesellschaft oft deutlich geringer sind [70]. Aber gerade Netzwerke in die Majoritätsgesellschaft, z. B. in Form von Nachbarschaftskontakten, scheinen sich nicht nur förderlich auf Interaktionen zwischen ethnisch diversen Gruppen auszuwirken, sondern auch Diskriminierung und Ausgrenzung entgegenzuwirken [57]. Darauf verweisen auch Forschungsergebnisse zu Vorurteilen, Stereotypen und psychischen Belastungen. Vorurteile gegenüber Minoritäten stellen Unterstützung und Solidarität infrage und können dementsprechend zu Diskriminierung und sozialer Isolation beitragen und damit psychische Gesundheit negativ beeinträchtigen [6, 43]. Zudem können in sozialen Interaktionen auftretende „ethnische" und rassistische Stereotype und Vorurteile, die sich gegen Minoritäten
Nervenarzt 2020 • 91:1017-1024

https://doi.org/10.1007/s00115-020-00990-1

○) Springer Medizin Verlag GmbH, ein Teil

von Springer Nature 2020

U. Kluge - M. C. Aichberger - E. Heinz -

C. Udeogu-Gözalan · D. Abdel-Fatah

\section{Rassismus und psychische Gesundheit}

\section{Zusammenfassung}

Der Beitrag gibt einen Überblick zu Rassismusdiskursen in Forschung und Praxis im Gesundheitsbereich und erörtert individuelle und institutionelle Auswirkungen von Rassismus und Diskriminierung auf die psychische Gesundheit. Daran anschließend wird erörtert, welche rassismuskritischen Transformationen in den Versorgungsstrukturen für psychisch erkrankte Personen notwendig sind, um eine gleichberechtigte Teilhabe von Menschen, die von Diskriminierung und Rassismus betroffen sind, zu ermöglichen.

\section{Schlüsselwörter}

Diskriminierung · Psychische Gesundheit . Individuelle Auswirkungen · Institutionelle Auswirkungen $\cdot$ Rassimus

\section{Racism and mental health}

\section{Abstract}

The article provides an overview of racism discourses in research and clinical practice in the health sector and discusses the individual and institutional effects of racism and discrimination on mental health. In addition, suggestions are provided as to which racism critical transformations in healthcare structures for mentally ill persons are necessary in order to enable equitable participation for people affected by discrimination and racism.

Keywords

Discrimination - Mental health · Individual effects · Institutional effects $\cdot$ Racism

richten, zu hohen psychischen Belastungen bei den davon betroffenen Personen führen [27, 42].

So zeigen Untersuchungen zum Psychoserisiko bei Migrant*innen erhöhte Psychoseraten in Nachbarschaften, in denen wenige andere Migrant*innen gleicher Herkunft lebten $[14,84]$. Hierbei ist weiterhin Gegenstand aktueller wissenschaftlicher Debatten, inwieweit Diskriminierung eine kausale oder mediieren- 
de Rolle spielt [80]. In jedem Fall kommt rassistischer Diskriminierung als sozialem Stressor eine zentrale Rolle in den betroffenen Gruppen zu, welcher das Risiko für eine psychotische Episode erhöht [83]. Auch neurobiologische Erkenntnisse zum Einfluss sozialen Stresses auf psychische Verarbeitungsprozesse könnten diese Hypothese stützten. So zeigte eine Reihe von Studien, dass sozialer Stress, z. B. in Form aversiver sozialer Ausgrenzung, die dopaminerge Neurotransmission verstärken kann [76, 77].

Dass die spezifische migrantische bzw. postmigrantische Lebenssituation einen Moderatoreffekt für den Zusammenhang von sozialer Ausschließung, Armut und psychischer Gesundheit haben kann [72], bestätigen neuere Studien zur psychischen Gesundheit Geflüchteter deutlich [15]. So konnte gezeigt werden, wie sich unsichere Lebensbedingungen, gesellschaftlicher Ausschluss und das Wohnumfeld auf psychische Belastungen auswirken [22, 39]. Gerade für Geflüchtete kommt noch eine weitere spezifische Ausschlusserfahrung - der unsichere Aufenthaltsstatus - hinzu, die wesentlichen Einfluss auf die psychische Gesundheit bei Geflüchteten hat [54].

\section{Rassismus und Diskriminierung in Versorgungsstrukturen}

Ethnokulturelle Minderheiten und Migrant*innen erfahren bei der Inanspruchnahme medizinischer Versorgung vielfach Diskriminierung [38]. Diese Erfahrungen können von subtilen Formen der Ausgrenzung bis hin zu expliziten Formen der Diskriminierung reichen. Sie manifestieren sich im direkten Kontakt mit anderen Patient ${ }^{*}$ innen ebenso wie mit dem Gesundheits- oder Servicepersonal. Studien zeigen, dass wahrgenommene Diskriminierung mit Verzögerungen bei der Suche nach einer Behandlung verbunden ist $[17,85]$, die die Motivation für Präventionsbemühungen senkt [37] und die Compliance gegenüber ärztlichen Empfehlungen beeinträchtigt [19]. Eine kürzlich in Deutschland durchgeführte Umfrage ergab, dass wahrgenommene ethnische Diskriminierung und Rassismus im Gesundheitswesen häufig mit sozialer
Diskriminierung verbunden war und dabei häufiger mit Herabsetzungen als mit explizitem Ausschluss von Gesundheitsdienstleistungen einhergeht [5].

Menschen erfahren in Krankenhäusern Diskriminierung allerdings nicht nur wegen ihrer Migrationsgeschichte, sondern auch weil ihre Lebensbedingungen mit anderen Dimensionen sozialer Ungleichheit verschränkt sind. Sie haben beispielsweise schlechtere Bildungschancen und häufig ein geringeres Einkommen, was wiederum zu Nachteilen im Gesundheitswesen führt [26, 60].

\section{) Corona macht soziopolitisch determinierte Gesundheitsrisiko- faktoren deutlich}

Dass Gesundheitsrisikofaktoren soziopolitisch determiniert sind, wurde auch im Zusammenhang mit der aktuellen Corona-Situation deutlich. Es zeigten sich auch hier die Auswirkungen von Diskriminierung und strukturellem Rassismus. Ähnlich wie bei der Influenzapandemie A (H1N1; [24, 74]) weisen zahlreiche Studien und national veröffentliche Statistiken auf eine stark erhöhte Infektions-, Hospitalisierungsund Sterberate durch COVID-19 bei Schwarzen und ethnischen Minderheiten im Vergleich zu Weißen in den USA und Großbritannien hin [4, 21, 32, 44, 56, 68]. Eine Reihe von Risikofaktoren, die von biologischen bis hin zu sozialen und systembedingten Elementen reicht, wurde als Haupterklärungsansätze für die Ungleichheiten bei den Gesundheitsfolgen von COVID-19 identifiziert [4, $32,34,56,71]$. So weisen Studien darauf hin, dass ethnische Minderheiten häufiger unter Herzkreislauferkrankungen, Diabetes, chronischen Lungenerkrankungen und Bluthochdruck leiden, die diese Gruppen einem höheren Risiko für eine schwere Folgeerkrankung durch COVID-19 auszusetzen [4, 32, 71]. Biologische Risikofaktoren können dabei nicht isoliert von anderen sozialen und strukturellen Faktoren betrachtet werden, die eine maßgebliche Auswirkung auf Krankheitsprävention und Gesundheitsfolgen haben [69]. Struk- turelle Ungleichheiten wie geringerer sozioökonomischer Status [71], beengte Wohnverhältnisse [56], höherer Beschäftigungsanteil in systemrelevanten Berufen ohne ausreichende Möglichkeiten zu „social distancing“ [63] sowie ein geringerer Anteil an Krankenversicherten $[21,34]$ erhöhen offenbar die Risiken für COVID-19-Erkrankungen und erschweren den Verlauf der Krankheit bei ethnischen Minderheiten, Schwarzen und anderen „people of color“.

\section{》) Es muss sich eine offene Diskussionskultur über den Abbau von Diskriminierung etablieren}

In den letzten Jahren haben die Forderungen nach einer interkulturellen Öffnung im deutschen Gesundheitswesen zugenommen und auch die Bemühungen zu deren Umsetzung sind verstärkt worden, um eine gleichberechtigte Teilhabe aller Gruppen und Communitys im System zu erreichen [67]. Allerdings ermöglicht dies nicht automatisch, diskriminierungs- und rassismusbedingte Ungleichheiten beim Zugang zu und in der Gesundheitsversorgung abzubauen. Für eine rassismus- und diskriminierungssensible Forschung und Versorgung bedarf es der Etablierung einer offenen Diskussionskultur über Diskriminierungserfahrungen und mögliche Wege zum Abbau von Diskriminierung und eines institutionellen Bewusstseins für diskriminierendes Verhalten. Nur in einem solchen Prozess können schrittweise und dauerhafte Änderungen der jeweiligen Praxen herbeigeführt werden. Wünschenswert wäre zukünftig eine intersektionale Perspektive, die eine Verschränkung der verschiedenen Dimensionen sozialer Ungleichheit und Ausschließungsprozesse berücksichtigt, um uns mit den vielfältigen Formen sozialer Benachteiligung und Mehrfachdiskriminierung auseinanderzusetzen. So können Menschen in ihrer Komplexität wahrgenommen und Verbindungen und Solidaritäten erleichtert werden.

Im Jahr 2017 erkannte die Weltgesundheitsorganisation (WHO) die 
Hier steht eine Anzeige.

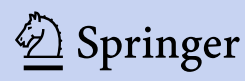


umfassenden Auswirkungen verschiedener Formen der Diskriminierung im Gesundheitswesen an und forderte ein Ende der Diskriminierung im Gesundheitswesen [86]. Zudem hat die WHO die Empfehlungen der UN befürwortet, die eine Priorisierung folgender Bereiche im Gesundheitswesen formulieren: Unterstützung der Beschäftigten im Gesundheitswesen, die zum Teil selbst von Diskriminierung betroffen sind, sodass sie ihre Rechte geltend machen können, die Entwicklung eines stabilen rechtlichen Rahmens und Antidiskriminierungsbemühungen sowohl im Gesundheitswesen als auch in anderen gesellschaftlichen Bereichen $\mathrm{zu}$ stärken.

\section{Fazit für die Praxis}

- „Rasse“ und „Rassen“ sind soziale

Konstruktionen, die keine biologi-

sche Entsprechung haben, sondern

menschengemachte Macht- und Do-

minanzkategorien repräsentieren.

Dabei ist Rassismus ein historisch

gewachsenes Phänomen, welches

sich $u$. a. in einer strukturellen Be-

nachteiligung bestimmter Gruppen

ausdrückt. Zu den davon betroffenen

Personengruppen gehören in be-

sonderem Maße Personen, die einer

ethnischen Minderheit angehören

bzw. einen Migrationshintergrund haben.

- Es wurde in vielen Studien doku-

mentiert, dass sich Exklusion und

Diskriminierung negativ auf die

psychische Gesundheit auswirken

können. Ausschlaggebend sind dabei nicht nur individuelle Diskriminierungserlebnisse, sondern in starkem Maße auch strukturelle Diskriminierung.

- Strukturelle Diskriminierung führt dazu, dass ethnische Minderheiten, „people of color" und Menschen mit Migrationshintergrund über die Lebensspanne hinweg zahlreichen indirekten und direkten Benachteiligungen ausgesetzt sind, die wir auch im Gesundheitswesen beobachten können.

\section{Korrespondenzadresse}

Prof. Dr. U. Kluge

Klinik für Psychiatrie und Psychotherapie, Charité Campus Mitte, Charité Universitätsmedizin Berlin

Charitéplatz 1, 10117 Berlin, Deutschland

ulrike.kluge@charite.de

\section{Einhaltung ethischer Richtlinien}

Interessenkonflikt. U. Kluge, M.C. Aichberger, E. Heinz, C.Udeogu-Gözalan und D. Abdel-Fatah geben an, dass kein Interessenkonflikt besteht.

Für diesen Beitrag wurden von den Autoren keine Studien an Menschen oder Tieren durchgeführt. Für die aufgeführten Studien gelten die jeweils dort angegebenen ethischen Richtlinien.

\section{Literatur}

1. ADS Antidiskriminierungsstelle des Bundes (Hrsg) (2015) Diskriminierung auf dem Wohnungsmarkt - Strategien zum Nachweis rassistischer Benachteiligungen von der Antidiskriminierungsstelle des Bundes. https:// www.antidiskriminierungsstelle.de/SharedDocs/ Downloads/DE/publikationen/Expertisen/ Expertise_Wohnungsmarkt_20150615.pdf? blob=publicationFile. Zugegriffen: 20. Juli 2020

2. Aichberger MC, Bromand Z, Rapp MA, Yesil R, Heredia Montesinos A, Temur-Erman S, Heinz A, Schouler-Ocak M (2015) Perceived ethnic discrimination, acculturation, and psychological distress in women of Turkish origin in Germany. Soc Psychiatry Psychiatr Epidemiol 50:1691-1700

3. APA (1996) Statement of biological aspects of race. Am JPhysiol Anthropol 101:569-570

4. Azar KMJ, Shen Z, Romanelli RJ, Lockhart SH, Smits K, Robinson S, Pressman AR (2020) Disparities in outcomes among COVID-19 patients in a large health care system in California. Health Aff. https://doi.org/10.1377/hlthaff.2020.00598

5. Beigang S, FetzK, KalkumD,Otto M(2017) Diskriminierungserfahrungen in Deutschland. Ergebnisse einer Repräsentativ- und Betroffenenbefragung. Antidiskrimiunierungsstelle des Bundes. Nomos, Baden-Baden

6. Benner AD, Wang $Y$, Shen $Y$, Boyle $A E$, Polk R, Cheng YP (2018) Racial/ethnic discrimination and well-being during adolescence: a meta-analytic review. Am Psychol 73(7):855-883. https://doi. org/10.1037/amp0000204

7. Benton MJ (2020) Cowen's history of life, 6. Aufl. Wiley-Blackwell, Hoboken

8. Berg AO, Melle I, Rossberg J, Romm KL, Larsson S, Lagerberg TV, Andreassen OA, Hauff E (2011) Perceived discrimination is associated with severity of positive and depression/anxiety symptoms in immigrants with psychosis: a crosssectional study. BMC Psychiatry 11:77

9. Berliner Institut für empirische Integrations- und Migrationsforschung (BIM), Forschungsbereich beim Sachverständigenrat deutscher Stiftungen für Integration und Migration (SVR-Forschungsbereich) (2017) Vielfalt im Klassenzimmer. Wie
Lehrkräfte gute Leistung fördern können. BIM Berlin

10. Bhui K, Stansfeld S, McKenzie K, Karlsen S, Nazroo J, Weich S (2005) Racial/ethnic discrimination and common mental disorders among workers: findings from the EMPIRIC Study of Ethnic Minority Groups in the United Kingdom. Am J Public Health 95:496-501

11. Bonefeld M, Dickhäuser $O$ (2018) (Biased) grading of students' performance: students' names, performance level, and implicit attitudes. Front Psychol. https://doi.org/10.3389/fpsyg.2018.00481

12. Bonilla-Silva E (1997) Rethinking racism: toward a structural interpretation. Am Sociol Rev 62(3):465-480

13. Bonilla-Silva E (2015) More than prejudice: restatement, reflections, and new directions in critical race theory. Sociol Race Ethn 1(1):73-87

14. Boydell J, van Os J, McKenzie K, Allardyce J, Goel R, McCreadie RG, Murray RM (2001) Incidence of schizophrenia in ethnic minorities in London: ecological study into interactions with environment. BMJ 323:1336

15. Brandt L, Henssler J, Müller M, Wall S, Gabel D, Heinz A (2019) Risk of psychosis among refugees: a systematic review and meta-analysis. Jama Psychiatry 76(11):1133-1140. https://doi.org/10. 1001/jamapsychiatry.2019.1937

16. Brown RA, Armelagos GJ (2001) Apportionment of racial diversity: a review. Evol Anthropol 10:34-40

17. Burgess DJ, Ding $Y$, Hargreaves $M$, van Ryn $M$, PhelanS(2008) The association between perceived discrimination and underutilization of needed medical and mental health care in a multiethnic community sample. J Health Care Poor Underserved 19:894-911

18. Cantor-Graae E, Selten JP (2005) Schizophrenia and migration: a meta-analysis and review. Am J Psychiatry 162:12-24

19. Casagrande S, Gary T, LaVeist TA, Gaskin DJ, Cooper LA (2007) Perceived discrimination and adherence to medical care in a racially integrated community. J Gen Intern Med 22:389-395

20. Cavalli-Sforza L, Cavalli-Sforza F (1994) Verschieden und doch gleich: Ein Genetiker entzieht dem Rassismus die Grundlage. Droemer Knaur, München (Aus dem Italienischen von Sylvia Höfer)

21. CDC (2020) Coronavirus Disease 2019 (COVID-19). https://www.cdc.gov/coronavirus/2019-ncov/ need-extra-precautions/racial-ethnic-minorities. html. Zugegriffen: 10. Juli 2020

22. Chu T, Keller AS, Rasmussen A (2013) Effects of post-migration factors on PTSD outcomes among immigrant survivors of political violence. J Immigr Minor Health 15:890-897

23. Cremer H (2012) „Rassismus"? Die Debatte zu Aussagen von Thilo Sarrazin hat verdeutlicht, wie eng der Begriff in Deutschland verstanden wird. In: Heinz A, Kluge U (Hrsg) Einwanderung - Bedrohung oder Zukunft? Mythen und Fakten zur Integration. Campus, Frankfurt/New York, S233-249

24. Dee DL, Bensyl DM, Gindler J, Truman BI, Allen BG, D'Mello T, Pérez A, Kamimoto $L$, Biggerstaff M, Blanton L, Fowlkes A (2011) Racial and ethnic disparities in hospitalizations and deaths associated with 2009 pandemic influenza $\mathrm{A}(\mathrm{H} 1 \mathrm{~N} 1)$ virus infections in the United States. Ann Epidemiol 21(8):623-630

25. Delgado R, Stefancic J (2001) Critical race theory. New York University Press, New York

26. Diehl C, Hunkler C, Kristen C (2016) Ethnische Ungleichheiten im Bildungsverlauf. Eine Einführung. In: Diehl C, Hunkler C, Kristen C (Hrsg) 
Ethnische Ungleichheiten im Bildungsverlauf. Mechanismen, Befunde, Debatten. Springer VS, Wiesbaden, S33-70

27. Elias A, Paradies Y (2016) Estimating the mental health costs of racial discrimination. BMC Public Health 16(1):1205. https://doi.org/10.1186/ s12889-016-3868-1

28. EPI (2019) Analysis of current population survey annual social and economic supplement historical poverty tables. https://www.epi.org/blog/racialand-ethnic-income-gaps-persist-amid-unevengrowth-in-household-incomes/.Zugegriffen: 10. Juli 2020

29. Fischer MS, Hossfeld U, Krause J, Richter S (2019) Jenaer Erklärung - Das Konzept der Rasse ist das Ergebnis von Rassismus und nicht dessen Voraussetzung. Biol Unserer Zeit 49(6):399-402

30. Ford C, Airhihenbuwa C (2010) The public health critical race methodology: praxis for antiracism research. Soc Sci Med 71:1390-1398

31. Fuchs B (2003) „Rasse“, „Volk”, Geschlecht. Anthropologische Diskurse in Österreich 1850-1960. Campus, Frankfurt/New York

32. Garg S, Kim L, Whitaker M, O'Halloran A, Cummings C, Holstein R, Fry A (2020) Hospitalization rates and characteristics of patients hospitalized with laboratory-confirmed coronavirus disease 2019-COVID-NET, 14 states, March 1-30, 2020. MMWR Morb Mortal Wkly Rep 69(15):458-464

33. Gomez L, Lopez N (Hrsg) (2013) Mapping race: critical approaches to health disparities research. Rutgers University Press, New Brunswick, NJ

34. Gregorio A, Millet GA, Jones AT, Benkeser D, Baral S, Mercer L, Beyrer Cetal (2020) Assessing differential impacts of COVID-19 on black communities. Ann Epidemiol 47:37-44. https://doi.org/10.1016/j. ann.epidem.2020.05.003

35. Guillaumin C (1992) Sexe, race et pratique du pouvoir. Paris: Editions Cote-femmes.

36. Harrell SP (2000) A multidimensional conceptualization of racism-related stress: implications for the well-being of people of color. Am JOrthopsychiatry 70:42-57

37. Hausmann LR, Jeong K, Bost JE, Ibrahim SA (2008) Perceived discrimination in health care and use of preventive health services. J Gen Intern Med 23:1679-1684

38. Hausmann LRM, Hannon MJ, Kresevic DM, Hanusa BH, Kwoh CK, Ibrahim SA (2011) Impact of perceived discrimination in healthycare on patient-provider communication. Med Care 49(7):626-633

39. Heeren M, Wittmann L, Ehlert U, Schnyder U, Maier T, Müller J (2014) Psychopathology and resident status-comparing asylum seekers, refugees, illegal migrants, labor migrants, and residents. Compr Psychiatry 55:818-825

40. Heinz A, Deserno L, Reininghaus U (2013) Urbanicity, social adversity and psychosis. World Psychiatry 12:187-197

41. Heinz A, Müller DJ, Kluge U (2018) Rassen und Rassismen. In: Machleidt W, Kluge U, Sieberer M, Heinz A (Hrsg) Praxis der Interkulturellen Psychiatrie und Psychotherapie. Migration und psychische Gesundheit. Elsevier, Urban \& Fischer, München, S93-102

42. Heinz A, Müller DJ, Krach S, Cabanis M, Kluge U (2014) The uncanny return of the race concept. Front Hum Neurosci. https://doi.org/10.3389/ fnhum.2014.00836

43. Henssler J, Brandt L, Muller M, Liu S, Montag C, Sterzer P, Heinz A (2019) Migration and schizophrenia: meta-analysis and explanatory framework. Eur
Arch Psychiatry Clin Neurosci. https://doi.org/10. 1007/s00406-019-01028-7

44. Holtgrave DR, Barranco MA, Tesoriero JM, Blog DS, Rosenberg ES (2020) Assessing racial and ethnic disparities using a COVID-19 outcomes continuum for New York State. Ann Epidemiol.https://doi.org/ 10.1016/j.annepidem.2020.06.010

45. Jones CP (2000) Levels of racism: a theoretic framework and a gardener's tale. Am J Public Health 90(8):1212-1215

46. Karlsen S, Nazroo J, McKenzie K, Bhui K, Weich S (2005) Racism, psychosis and common mental disorderamongethnicminority groupsin England. Psychol Med 35:1795-1803

47. Kirmes M, Barwinski R (Hrsg) (2020) Schwerpunktheft: Opfer rechter Gewalt. Trauma-Zeitschrift für Psychotraumatologie und ihre Anwendung, Bd. 18(1). Asanger, Kröning

48. Kluge U, Bostanci S (2012) MigrantInnen als Bedrohung - Die neue Diskursfähigkeit einst abgelegter Weltbilder. In: Heinz A, Kluge U (Hrsg) Einwanderung - Bedrohung oder Zukunft? Mythen und Fakten zur Integration. Campus, Frankfurt/New York, S 16-35

49. Koller C (2015) Was ist eigentlich Rassismus? Bundeszentrale für politische Bildung, (8. Dezember 2015). https://www.bpb.de/politik/extremismus/ rechtsextremismus/213678/was-ist-eigentlichrassismus. Zugegriffen:20. Juli 2020

50. Koopmans R, Veit S, Yemane R (2018) Ethnische Hierarchien in der Bewerberauswahl: Ein Feldexperiment zu den Ursachen von Arbeitsmarktdiskriminierung. WZB Discussion Paper, No. SP VI 2018-104. Wissenschaftszentrum Berlin für Sozialforschung (WZB), Berlin

51. Krause J (2019) Die Reise unserer Gene: Eine Geschichte über uns und unsere Vorfahren - mit Thomas Trappe. Propyläen, Berlin

52. Krieger N (1999) Embodying inequality: a review of concepts, measures, and methods for studying health consequences of discrimination. Int JHealth Serv 29:295-352

53. Krieger N (2012) Methods for the scientific study of discrimination and health: an ecosocial approach. Am J Public Health 102:936-944

54. Lamkaddem M, Essink-Bot ML, Devillé W, Gerritsen A, Stronks K (2015) Health changes of refugees from Afghanistan, Iran and Somalia: the role of residence status and experienced living difficulties in the resettlement process. Eur J Public Health 25:917-922

55. Landrine $\mathrm{H}$, Klonoff EA, Corral I, Fernandez $\mathrm{S}_{\text {, }}$ Roesch S (2006) Conceptualizing and measuring ethnic discrimination in health research. J Behav Med 29:79-94

56. Lassale C, Gaye B, Hamer M, Gale CR, Batty GD (2020) Ethnic disparities in hospitalisation for COVID-19 in England: The role of socioeconomic factors, mental health, and inflammatory and pro-inflammatory factors in a community-based cohort study. Brain Behav Immun. https://doi.org/ 10.1016/j.bbi.2020.05.074

57. Laurence J (2011) The effect of ethnic diversity and community disadvantage on social cohesion: a multi-level analysis of social capital and interethnic relations in UK communities. Eur Sociol Rev 27:70-89

58. Link B, Phelan J (1995) Social conditions as fundamental causes of disease. J Health Soc Behav. https://doi.org/10.2307/2626958

59. Livingston FB (1962) On the non-existence of human races. Curr anthropol 3(3):279-281

60. Lokhande M (2016) Doppelt benachteiligt? Kinder und Jugendliche mit Migrationshintergrund im deutschen Bildungssystem. SVR-Forschungsbereich, Berlin

61. Ludwig J, Duncan GJ, Gennetian LA, Katz LF Kessler RC, Kling JR, Sanbonmatsu L (2012) Neighborhood effects on thelong-termwell-being of low-income adults. Science 337:1505-1510

62. Marmot M, Bell R (2016) Social inequalities in health: a proper concern of epidemiology. Ann Epidemiol 26:238-240

63. Mora GC, Schickler E (2020) Racial minorities at risk in the workplace and the economy. Berkeley IGS poll. https://escholarship.org/content/ qt1kd7h0sc/qt1kd7h0sc.pdf. Zugegriffen: 19. Juli 2020

64. Nadal KL, Griffin KE, Wong Y, Hamit S, Rasmus M (2014) The impact of racial microaggressions on mental health: counseling implications for clients of color. J Couns Dev 92(1):57-66

65. Nazroo JY (2003) The structuring of ethnic inequalities in health: economic position, racial discrimination, and racism. Am J Public Health 93:277-284

66. Pascoe EA, Richman SL (2009) Perceived discrimination and health: a meta-analytic review. Psychol Bull 135:531-554

67. Penka S, Faißt H, Vardar A, Borde T, Mösko MO Dingoyan D, Schulz H, Koch U, Kluge U, Heinz A (2015) Der Stand der interkulturellen Öffnung in der psychosozialen Versorgung - Ergebnisse einer Studie in einem innerstädtischen Berliner Bezirk. Psychother Psychosom Med Psychol 65(09/10):353-362

68. PHE (2020) Disparities in the risk and outcomes from COVID-19. https://assets.publishing.service. gov.uk/government/uploads/system/uploads/ attachment_data/file/892085/disparities_review. pdf.Zugegriffen: 19. Juli 2020

69. Phelan JC, LinkBG, Tehranifar P (2010) Social conditions as fundamental causes of health inequalities: theory, evidence, and policy implications. J Health SocBehav 51(1):28-40

70. Putnam RD (2007) E pluribus unum: diversity and community in the twenty-first century. The 2006 Johan Skytte Prize Lecture. Scand Pol Studs 30(2):137-174

71. Raifman MA, Raifman JR (2020) Disparities in the population at risk of severe illness from COVID19 by race/ethnicity and income. Am J Prev Med 59(1):137-139. https://doi.org/10.1016/j.amepre. 2020.04.003

72. Rapp MA, Kluge U, Penka S, Vardar A, Aichberger MC, Mundt AP, Schouler-Ocak M, Mösko M, Butler J, Meyer-Lindenberg A, Heinz A (2015) When local poverty is more important than your income: mental health in minorities in inner cities. World Psychiatry 14(2):249-250. https://doi.org/ $10.1002 / w p s .20221$

73. Rosenberg NA (2011) A population-genetic perspective on the similarities and differences among worldwide human populations. Hum Biol 83(6):659-684

74. Sachedina N, Donaldson LJ (2010) Paediatric mortality related to pandemic influenza $\mathrm{A} \mathrm{H} 1 \mathrm{~N} 1$ infection in England: An observational populationbased study. Lancet 27(376):1846-1852

75. Saini A (2019) Superior: the return of race science. Beacon, Boston

76. Selten JP, Cantor-Graae E (2005) Social defeat: risk factor for schizophrenia? Br J Psychiatry 187:101-102

77. Tidey JW, Miczek KA (1996) Social defeat stress selectively alters mesocorticolimbic dopamine release: an in vivo microdialysis study. Brain Res 721:140-149 
78. Tishkoff SA, Dietzsch E, Speed W et al (1996) Global patterns of linkage disequilibrium at the CD 4 locus and modern human origins. Science 271:1380-1387

79. VBRG (2020) Rechte, rassistische und Antisemitische Gewalt in Deutschland 2019 - Jahresbilanzen der Opferberatungsstellen. https:// www.verband-brg.de/rechte-rassistische-undantisemitische-gewalt-in-deutschland-2019jahresbilanzen-der-opferberatungsstellen/\# pressemitteilung. Zugegriffen: 20 . Juli 2020

80. Veling W, SusserE (2011b) Migration and psychotic disorders. Expert Rev Neurother 11:65-76

81. Veling W, HoekHW, Mackenbach JP (2008a) Perceived discrimination and the risk of schizophrenia in ethnic minorities: a case-control study. Soc Psychiatry Psychiatr Epidemiol 43:953-959

82. Veling W, Hoek HW, Selten JP, Susser E (2011a) Age at migration and future risk of psychotic disorders among immigrants in the Netherlands: a 7-year incidence study. Am J Psychiatry 168:1278-1285

83. Veling W, Pot-Kolder R, Counotte J, van Os J, van der Gaag M (2016) Environmental social stress, paranoia and psychosis liability: a virtual reality study. Schizophr Bull 42:1363-1371

84. Veling W, Susser E, van Os J, Mackenbach JP, Selten JP, Hoek HW (2008b) Ethnic density of neighborhoods and incidence of psychotic disorders among immigrants. Am J Psychiatry 165:66-73

85. Wamala S, Merlo J, Boström G, Hogstedt C (2007) Perceived discrimination, socioeconomic disadvantage and refraining from seeking medical treatment in Sweden. J Epidemiol Community Health 6:409-415

86. WHO (2017) Ending discrimination in health care settings. WHO, Genf

87. William DR, Williams-Morris R (2000) Racism and mental health: the African American experience. Ethn Health 5:243-268

88. Zeit-Online (2018) Erstochen, erschlagen, verbrannt. Todesopfer rechter Gewalt. https://www. zeit.de/gesellschaft/zeitgeschehen/2018-09/ rechtsxtremismus-todesopfer-gewalt-verdacht Zugegriffen:20. Juli 2020

\section{Mönter Norbert, Heinz Andreas, Utsch Michael Religionssensible Psychotherapie und Psychiatrie Basiswissen und Praxis- Erfahrungen.}

Stuttgart: Kohlhammer-Verlag 2020, 246 S., 35,00 EUR

(Fortsetzung von S. 1016)

Die religiöse Verantwortung des Individuums ("taklif") ist sowohl an die Geschlechtsreife (,'bulug") als auch an den Verstand (,'aql") gekoppelt und unterstreicht, dass das "Selbst" im Koran als dynamisches Konzept verstanden wird. Psychische Erkrankung wird im Arabischen häufig mit "gunun" umschrieben; eigenartiger Weise wird der gleiche Begriff verwendet, wenn es um die Abwehr von schmähenden Vorwürfen gegen den Propheten geht, was also auf eine eher negative Konnotation des Begriffes schließen lässt.

Die Existenz von „Ginns" (S. 106) als "Geistwesen" wird laut strenger Koranauslegung zwar verneint, spielt aber noch immer eine große Rolle, wenn es um die Erklärung schwer fassbarer somatischer und vor allem psychischer Beschwerden geht. Die dahintersteckende Abwehr von Stigmatisierungsängsten durch projektive Verschiebung auf übernatürliche Kräfte ist sicher kein vorrangiges Phänomen von muslimischen Patientinnen und Patienten, trotzdem sollte ein gewisses Grundwissen darüber vorhanden sein. Denn nur dann wird man „verstehen", dass diese "Djinnen" - eine ebenfalls verwendete Schreibweise (S. 184) - Menschen- oder Tier-förmige Gestalt annehmen und beim "Stochern in Müllhaufen“, „nächtlichen Besuchen von Friedhöfen“ ',Ausschütten von Schmutzwasser", "Intimkontakten ohne entsprechendes Reinigungsritual" etc. ihre unheilvolle Be-

setzung von Menschen vornehmen können. Die daraus resultierenden „präventiven“ wie "therapeutischen" Maßnahmen in Form von Gegenflüchen, Handauflegungen, Beschwörungen oder dem Tragen von Amuletten haben für viele Muslime weiterhin eine große Bedeutung.

Dr. Norbert Mönter hat in Berlin bereits 2003 den Arbeitskreis "Religion und Psychiatrie" gegründet, um angesichts der zunehmenden religiösen Pluralität der Stadtbevölkerung eine konstruktive Austausch- und Begegnungskultur zu etablieren. Das daraus hervorgehende PIRA-Projekt (PsychiatrieInformation-Religion-Austausch) hat zu einer äußerst fruchtbaren Zusammenarbeit mit Imamen und anderen Religionsvertretern in Berlin geführt, um eine bessere und differenziertere Unterscheidung von psychischen und religiös-spirituellen" Problemen zu ermöglichen. Von den 12 PIRA-Akteuren bezeichnen sich die 4 türkisch- und die 5 arabisch-stämmigen psychiatrischen und psychotherapeutischen Profis als „überwiegend engagiert gläubige Muslime“, von den 3 Deutschen gibt es hierzu keine Aussage, was in diesem Kontext nicht ganz untypisch sein dürfte. Und doch scheint der Kant'sche Spruch von der "Unhintertreiblichkeit des Transzendentalen“ weiterhin Gültigkeit zu haben, denn trotz des Rückganges der kirchlich organisierten Religiosität gibt es einen anhaltenden Zulauf bei esoterischen und außerkirchlichen Strömungen. Und gleichzeitig mehren sich wissenschaftlich fundierte Daten, dass eine überzeugt gelebte und rational dosierte Religiosität nicht nur eine Ressource sondern vor allem bei chronischen Erkrankungen einen Copingvorteil darstellt. Sowohl die Gefahren einer fehlgeleiteten Sinn- und Haltsuche durch missverstandene Dogmen als auch die positiven Bewältigungskräfte einer gesunden Gläubigkeit müssen ein sehr ernstes Anliegen für alle psychiatrisch-psychotherapeutisch Tätigen sein! Dieses Buch von Mönter, Heinz und Utsch ist in dieser Hinsicht eine ungeheure Bereicherung und darf in keinem Behandlungszimmer mit oder ohne Couch - fehlen!

Prof. Dr. med. Josef Bäuml 